\title{
Epiphrenic esophageal diverticulum within a leiomyoma: A rare cause of dysphagia
}

\author{
Omar Abdel-1ah Fernández ${ }^{1 *}$, Alberto Alvarez Delgado ${ }^{2}$, Felipe Carlos Parreño ${ }^{1}$ and Antonio Rodriguez ${ }^{2}$ \\ ${ }^{1}$ Gastro-esophageal Pathology Unit of the Department of Surgery and Digestive System, University Hospital of Salamanca, Spain \\ ${ }^{2}$ Endoscopy Unit of the Department of Digestive System, University Hospital of Salamanca, Spain
}

\begin{abstract}
Introduction: The diagnosis of symptomatic epiphrenic esophageal diverticulum is rare (15-20\%). Esophageal leiomyoma and epiphrenic diverticula are both uncommon, but may rarely occur together. In this patient a leiomyoma was found within an epiphrenic diverticulum.

Methods: We report the case of a 58-year-old man referred to our unit with a 4-year history of progressive and intermittent dysphagia, mainly to solids, and weight loss. Barium swallow revealed a distal esophageal stenosis secondary to a diverticulum; upper endoscopy showed an extrinsic submucosal compression of the cardia; and CT scan showed an infradiaphragmatic saccular dilation of the esophagus with a thickened wall. The patient was diagnosed with distal esophageal diverticulum with a submucosal tumor inside. Laparoscopic hiatal diverticulectomy including tumor, cardiomyotomy and a partial fundoplication was performed. Our objective is to discuss the diagnosis and treatment of this disease.
\end{abstract}

Results: The pathological study confirmed a leiomyoma of $5 \times 4 \mathrm{~cm}$ in an esophageal diverticulum. On the 3rd postoperative day, barium swallow confirmed good esophageal passage and gastric emptying. The patient was discharged on day 4. In the follow-up monitoring after one month and one year the patient was asymptomatic with good quality of life.

Discussion: Traditionally, treatment for an epiphrenic diverticulum consists of a diverticulectomy with or without myotomy, performed through a thoracotomy. Hiatal laparoscopy obviates the need for thoracotomy and placement of a chest tube. It would also allow an easier fundoplication after myotomy and diverticulectomy. This case has several unique aspects. Firstly, an esophageal diverticulum with a leiomyoma inside, which is in a subdiaphragmatic location; and secondly, the fact that it is not associated with primary motor disorder. Here, the leiomyoma causes the epiphrenic diverticulum.

Conclusion: Whenever a submucosal tumor within the diverticulum is present careful consideration should be given to the possibility of an associated neoplasm. We suggest that the majority of epiphrenic diverticula of the distal esophagus can be treated successfully by laparoscopic approach, which makes it possible to remove the associated tumor; and that it is at least as safe and effective as conventional transthoracic access but with the advantages of an early postoperative recovery.

\section{Introduction}

Esophageal leiomyoma and epiphrenic diverticula are both uncommon, but may rarely occur together. The epiphrenic diverticula are found in the last $10 \mathrm{~cm}$ of the esophagus; they represent $10 \%$ of all esophageal diverticula, with a radiological prevalence of $0.015 \%$ in the US and up to $2 \%$ in Europe [1], although the actual prevalence is unknown. They are usually the result of a pulsion mechanism, originated by an alteration of the esophageal motility [1-3]. The most frequently associated cause is achalasia, although it also has been identified in patients with diffuse esophageal spasm, symptomatic esophageal peristalsis and less often with peptic strictures and tumors [2]. Leiomyoma is the most common benign esophageal tumor, and yet it is 50 times less frequent than esophageal carcinoma. Their incidence ranges from $0.005 \%$ to $5 \%$ [3], and they only represent $0.4 \%$ of all esophageal tumors. $50 \%$ of them are located in the distal esophagus and usually intramurally [4].

Intradiverticular neoplasms are rare, and most are squamous cell carcinomas. A few cases have been published of leiomyoma $[5,6]$, malignant fibrous histiocytoma and metastatic neuroma [7]. There is little scientific literature on this topic and multiple controversies exist regarding the management of this disease.

\section{Clinical case}

We present the case of a 58-year-old man, without toxic habits and a medical history of malaria, trigeminal neuralgia, total thyroidectomy, and parathyroid resection for papillary cancer, hypothyroidism, and postoperative hypoparathyroidism.

He reports a 4-year history of dysphagia, first intermittent and then progressive, both to solid and liquids, exacerbated in the last 6 months, and associated with regurgitation and with loss of $10 \mathrm{~kg}$ of weight, without associated vomiting. He has not had previous episodes of aspiration or respiratory infections.

The findings on the physical examination were within normal

Correspondence to: Dr. Omar Abdel-lah Fernández, MD, Gastro-esophageal Pathology Unit of the Department of Surgery and Digestive System, University Hospital of Salamanca, C/Muñoz Torrero 1 5B, 37007 Salamanca. Spain, Tel: +34 923261198; E-mail: omarabdellah@gmail.com

Key words: esophageal diverticulum, epiphrenic diverticulum, leiomyoma of esophagus

Received: August 30, 2015; Accepted: September 19, 2015; Published: September 22, 2015 
limits. Laboratory parameters, hematological and biochemical tests were also normal.

Given this history, we first requested a barium swallow, which defined a saccular image of $5 \mathrm{~cm}$ with uniform walls in the distal esophagus at the level of the esophageal-stomach junction (Figure 1). Stenosis was observed. The upper endoscopy revealed an extrinsic compression that altered the cardia without mucosal ulceration.

Since the result of the tests was still unclear, we completed the work with a CT scan of the thorax and abdomen which showed a $5 \times 5 \mathrm{~cm}$ infradiaphragmatic dilatation of the esophagus with thickened walls of $2.5 \mathrm{~cm}$ and difficulties in contrast passage through the esophagus (Figure 2). Esophageal endoscopic ultrasound (EUS) revealed a concentric multilobular thickening of the muscular layer. PAAF was inconclusive. Esophageal manometry showed $30 \%$ of normal peristaltic waves associated to ineffective peristaltic waves, aperistaltic periods and hypertonic distal esophagus. These findings ruled out motor disorders such as achalasia or diffuse esophagus spasm. In conclusion, manometry showed vigorous peristalsis secondary to a mechanical obstructive problem.

The diagnosis was dysphagia caused by an infradiaphragmatic esophagus diverticulum within a submucosal tumor.

With this information the intervention was carried out. In the operating room we found a tumor of $5 \mathrm{~cm}$ in diameter on the left side of the abdominal esophagus, close to the esophageal gastric junction (Figure 3). A diverticulectomy was performed and the submucosal tumor was removed with Endo GIA $45 \mathrm{~mm}$ blue reloads (Echelom Ethicon Endo-Surgery, USA) after the placement of a 55 FR catheter in the esophageal lumen, extramucosal cardiomyotomy and anterior (Dor) fundoplication with laparoscopy (Figure 4). Intraoperative

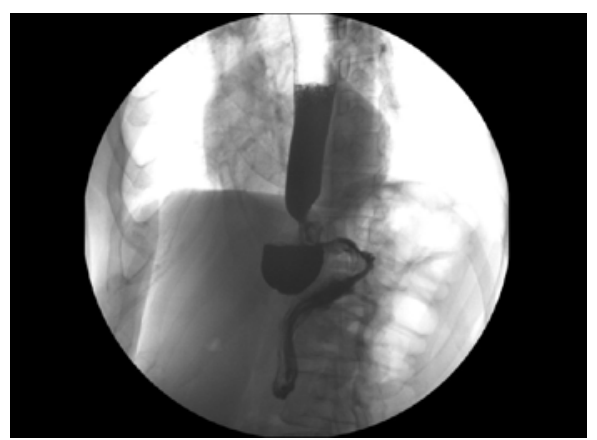

Figure 1. Saccular image with regular edges of $5 \mathrm{~cm}$ in the distal esophagus, with air-fluid level inside.

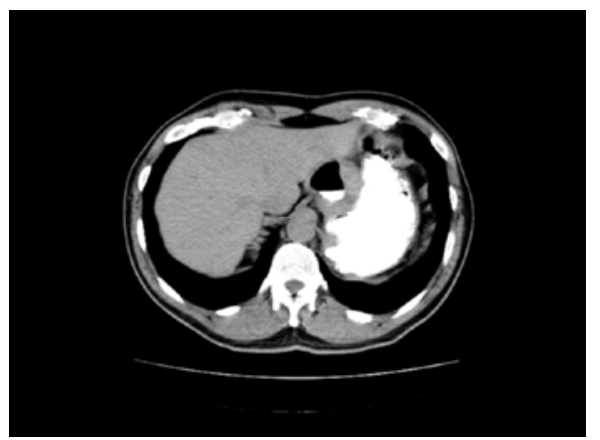

Figure 2. CT reveals a saccular formation with contrast and wall thickening.

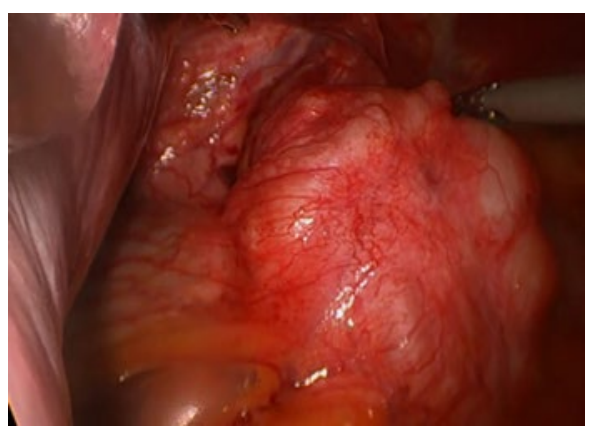

Figure 3. Intraoperative view of the tumor.

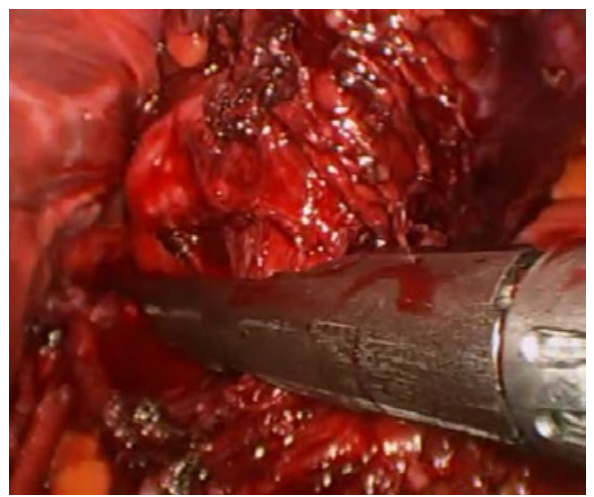

Figure 4. Section with Endo GIA of the neck of the diverticulum.

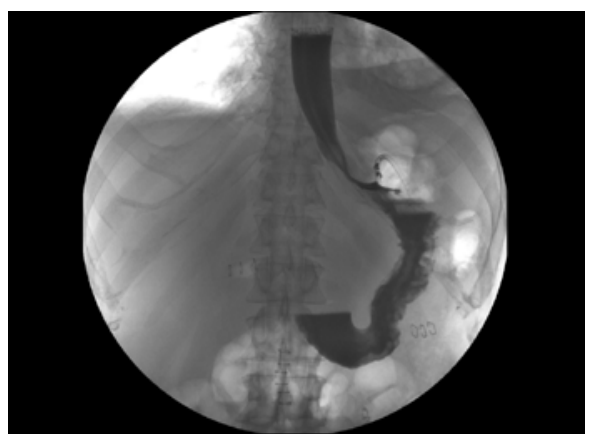

Figure 5. Postoperative control swallow.

endoscopy confirmed a complete diverticulectomy without stenosis or perforation of the mucosa.

\section{Results}

On the $3^{\text {rd }}$ postoperative day, Gastrografin ${ }^{\circledR}$ swallow showed good passage through the esophageal gastric junction and good gastric emptying (Figure 5). The patient initiated oral tolerance after that and he was discharged on the $4^{\text {th }}$ postoperative day.

The results of the histopathological study confirmed the existence of a $5 \times 4 \mathrm{~cm}$ multilobular leiomyoma which did not infiltrate the mucosa inside a mechanical esophageal diverticulum of $6 \times 4 \mathrm{~cm}$ with partially collapsed lumen associated with intense inflammation.

Immunohistochemistry: Actin +, Desmin +, CD34 and DOG-1, c-Kit-, S-100. 0/10HPF mitosis and Ki-67 index $<1 \%$.

In the follow-up session after 1 month the patient was asymptomatic 
with good oral tolerance, and after 12 months he had gained weight and had good quality of life.

\section{Discussion}

Leiomyoma is the most common benign tumor of the esophagus, but it is still rare; approximately $50 \%$ of them are asymptomatic. Seremetis et al. reviewed 838 cases of esophageal leiomyoma and less than 20 cases were associated with esophageal diverticula [8], but the authors did not specify how many of these were epiphrenic.

Diagnostic problems often arise, such as the fact that smooth muscle tumors may mimic mediastinal neoplasms, cysts, or even aneurysms; or complicate coexisting hiatal hernia and esophageal diverticula. Leiomyoma and carcinoma should be ruled out in the diagnosis of esophageal diverticulum.

The epiphrenic diverticulum is located in the last $10 \mathrm{~cm}$ of the esophagus. The origin of epiphrenic diverticula is uncertain, and most authors suggest that it results from an underlying alteration of the motility. The idea that an epiphrenic diverticulum is a consequence of a motor disorder is generally accepted [2]. Thomas et al. [9] reported in a 2001 study with 356 patients that $78 \%$ of them had a motor disorder. However, the case we report is an exception; we suggest that the diverticulum resulted from a retraction of the esophageal wall related to the extraluminal growth of the leiomyoma. It conditioned a distal mechanical obstruction; without associated specific motor disorder. The pathologic specimen showed a leiomyoma with normal esophageal mucosa and a true diverticulum containing all the layers of the esophageal wall.

Diagnostic management seems to be well established, barium swallow being the most useful initial exam. Esophagoscopy and esophageal manometry are important in the preoperative assessment, in order to exclude other pathologies. Other useful but non-routine tests are the CT scan of the thorax and abdomen, EUS and pH studies.

In the present case, given the particular characteristics of the condition, we performed a CT scan of thorax and abdomen and EUS for the evaluation of the associated tumor.

The indication of surgery in cases of asymptomatic diverticula is controversial, while its indication in symptomatic cases seems clear, as in our case, and even more so if a submucosal tumor is associated [10].

Nowadays, the development of minimally invasive surgery provides a better view of the distal esophagus through a hiatal approach, which allows a better exposure of the lesion that will facilitate the angle of approach for the endoscopic stapler, parallel to the esophagus, which is considerably more difficult in the thoracoscopic approach. It also facilitates an extended myotomy in the cardia and fundoplication. Other widely accepted advantages are the faster recovery of the patient and, as a result, the reduction of the patient's length of hospital stay compared with the thoracoscopic approach [11-13].

Limitations of the laparoscopic approach are described in middle esophageal diverticula and in cases with extensive inflammatory reaction $[1,11,12]$.

Historically, the conventional treatment of the epiphrenic diverticulum consists of diverticulectomy with or without myotomy with thoracotomic approach, which allowed exposure of the diverticula. Nowadays, the standard surgical approach for the majority of authors combines diverticulectomy, esophageal cardiomyotomy and antireflux technique; mainly through hiatal laparoscopic access $[1,3,11$ -
14]. Currently, controversies exist on several aspects. Regarding cardiomyotomy, most authors consider it necessary, especially if there is an associated motor alteration, which coincides with our view. With regard to the type of fundoplication, there seems to be a widespread consensus opinion in the literature that it should be partial. As we said before, in our case, an anterior Dor type fundoplication was performed. We believe that it may satisfactorily control the postoperative gastroesophageal reflux and prevent the serious consequences of a suture dehiscence.

This paper shows an extremely rare case, with several remarkable aspects, such as the presence of leiomyoma within a diverticulum, which requires more preoperative studies that would not be routine in conventional esophageal diverticula and which should be taken into account in the clinical practice. In our patient, we completed the tests with EUS, biopsy, pH studies and a CT scan. Another relevant aspect is that it was not associated with a primary motor disorder. In our case, the epiphrenic diverticulum was triggered by the leiomyoma. The last remarkable aspect is the location of the lesion in the abdominal esophagus, which makes it almost essential to use the laparoscopic approach.

\section{Conclusion}

When a submucosal tumor is present within the diverticulum, careful consideration should be given to the possibility of an associated neoplasm.

We believe that most epiphrenic diverticula of the distal esophagus can be treated successfully by laparoscopic approach, allowing removal the associated tumor; and that it is at least as safe and effective as conventional transthoracic access, but with the advantages of an early postoperative recovery.

\section{References}

1. Kilic A, Schuchert MJ, Awais O, Luketich JD, Landreneau RJ (2009) Surgical management of epiphrenic diverticula in the minimally invasive era. JSLS13: 160-164. [Crossref]

2. Nehra D, Lord RV, DeMeester TR, Theisen J, Peters JH, et al. (2002) Physiologic basis for the treatment of epiphrenic diverticulum. Ann Surg235: 346-354. [Crossref]

3. Ruiz De Angulo Martín D, Ortiz Escandell MA, Martínez De Haro LF, Munítiz Ruiz V, ParrillaParicio P (2009) Epiphrenic diverticula: when and how to operate? Cir Esp85: 196-204. [Crossref]

4. Priego P, Lobo E, Alonso N, Gil Olarte MA, Pérez de Oteyza J, et al. (2006) Surgical treatment of esophageal leiomyoma: an analysis of our experience. Rev EspEnferm Dig98: 350-358. [Crossref]

5. Hamilton S (1988) Esophageal leiomyoma arising in an epiphrenic diverticulum. Eur J Radiol8: 118-119. [Crossref]

6. Hodge GB (1970) Esophageal leiomyoma associated with an epiphrenic diverticulum and hiatus hernia. Am Surg36: 538-543.[Crossref]

7. Smith GS, Isaacson JR, Dempsey MB, Falk GL (2001) Laparoscopic excision of esophageal leiomyoma through an anterior esophagotomy. Dis Esophagus 14: 278-279. [Crossref]

8. Seremetis MG, Lyons WS, deGuzman VC, Peabody JW Jr (1976) Leiomyomata of the esophagus. An analysis of 838 cases. Cancer38: 2166-2177. [Crossref]

9. Thomas ML, Anthony AA, Fosh BG, Finch JG, Maddern GJ (2001) Oesophageal diverticula. Br J Surg88: 629-642. [Crossref]

10. Zaninotto G, Portale G, Costantini M, Zanatta L, Salvador R, et al. (2011) Therapeutic strategies for epiphrenic diverticula: systematic review. World J Surg35: 1447-1453 [Crossref]

11. Soares RV, Montenovo M, Pellegrini CA, Oelschlager BK (2011) Laparoscopy as the initial approach for epiphrenic diverticula. SurgEndosc25: 3740-3746. [Crossref] 
12. Fisichella PM, Pittman M, Kuo PC (2011) Laparoscopic treatment of epiphrenic diverticula: preoperative evaluation and surgical technique. How I do it. $J$ GastrointestSurg15: 1866-1871. [Crossref]

13. Motoyama S, Maruyama K, Okuyama M, Sasaki K, Sato Y, et al. (2006) Laparoscopic long esophagomyotomy with Dor's fundoplication using a transhiatal approach for an epiphrenicesophageal diverticulum. Surg Today36:758-760. [Crossref]

14. Hoshino M, Omura N, Yano F, Tsuboi K, Matsumoto A, et al. (2010) Laparoscopic Heller myotomy and Dor fundoplication combined with laparoscopic diverticular introversion suturing for achalasia complicated by epiphrenic diverticulum: report of a case. Surg Today40:158-161. [Crossref]

Copyright: (C) 2015 Abdel-lah Fernández O. This is an open-access article distributed under the terms of the Creative Commons Attribution License, which permits unrestricted use, distribution, and reproduction in any medium, provided the original author and source are credited. 